\title{
三值 $b_{j}$ 图 及 其 应用 \\ 胡，铮浩 \\ (s) $m \times$ क
}

由于人以视觉形式感受大部分信息, 所以图形表示及图形处理方法被广泛采用于各类问 题的研究中. 逻辑代数的研究也同样如此.

在二值逻辑中, 采用的图形有 $K$ 图 ${ }^{[1]}, b_{i}$ 系数图 ${ }^{[2]}$ 及 $R_{i}$ 谱系数图 ${ }^{[3]}$. 在多值逻辑的研究 中, 往往推广二值逻辑中的研究方法。然而, 由于多值造辑的复杂性, 至今还仅局限于多值 $K$ 图. 本文旨在提出三值 $b_{j}$ 系数图, 并介绍其在逻辑综合中的应用, 为多值逻辑的研究补充一 种图形处理手段。

\section{一、三值 $\boldsymbol{b}_{i}$ 系数图}

与文献 [2] 相仿, 三值 $b_{i}$ 系数图相应于逻辑函数的规范 RM 展开式.

任意 $n$ 变量三值函数的规范 RM 展开式可写成:

$$
\begin{aligned}
& f\left(X_{1}, \cdots, X_{n}\right)=b_{0} X_{1}^{0} X_{2}^{0} \cdots X_{n-1}^{0} X_{n}^{0} \oplus b_{1} X_{1}^{0} X_{2}^{0} \cdots X_{n-1}^{0} X_{n}^{1} \oplus b_{2} X_{1}^{0} X_{2}^{0} \cdots X_{n-1}^{0} X_{n}^{2} \\
& \oplus \cdots \oplus b_{i} X_{1}^{i_{1}} X_{2}^{t_{2}} \cdots X_{n}^{n} \oplus \cdots \oplus b_{3}^{n}{ }_{-1}^{n} X_{1}^{2} X_{2}^{2} \cdots X_{n}^{2},
\end{aligned}
$$

式中 $X_{k}^{0}-1, X_{k}^{1}=X_{k}(k=1,2, \cdots, n), b_{i} \in\{0,1,2\}\left(j=0,1, \cdots, 3^{n}-1\right), e_{k} \in\{0$, $1,2\}(k-1,2, \cdots, n)$. 展开式中任一积项的系数 $b_{i}$ 的下标由该积项各变量的指数 $\left(e_{k}\right)$ 组成的三进制数给出, 即

$$
(j)_{10}=\left(e_{1} e_{2} \cdots e_{k} \cdots e_{n}\right)_{3},
$$

将式 (1) 中的 $3^{n}$ 个系数 $\left(b_{i}\right)$ 按一定规律 (地址) 填人框图, 就构成三值 $b_{i}$ 系数图. 图 1(a), (b) 和 (c) 分别给出单变量、二变量和三变量三值函数的三值 $b_{i}$ 系数图结构. 图中框外数字 $(0,1,2)$ 表示与所标变量相应的地址, 即式 (1) 各积项中各变量的指数 $e_{k}$ 。 在由 $n$ 个地址决 定的方格中填入式 (1) 中相应变量积项的系数.

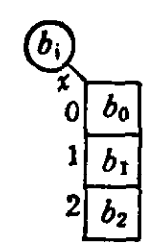

(a)

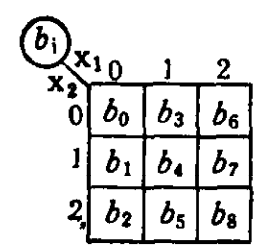

(b)

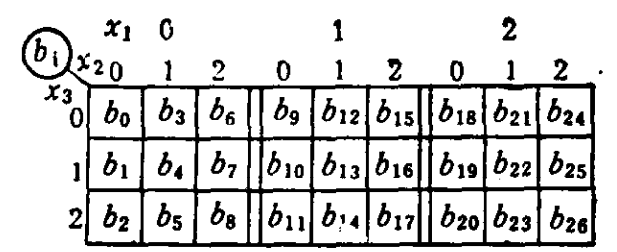

(c)

图 1 三值 $b$, 系数图结构

(b) 中的 $b_{1}$, 根据式 (2), 有 $(7)_{10}=(21)_{3}$, 相应变量积项是 $X_{1}^{2} X_{2}^{1}$, 图中 $b_{7}$ 方格的地垃正是 $e_{1}=2, c_{2}=1$;

(c) 中的 $b_{16}$, 根据式 (2), 有 $(16)_{10}=(121)_{3}$, 相应变最积项是 $X_{1}^{1} X_{2}^{2} X_{\mathrm{s}}^{1}$, 因中 $b_{16}$ 方格的地址正是 $e_{1}=1, c_{2}=2, c_{3}=1$ 
应该指出两点: (1) 绘制三值 $b_{i}$ 系数图, 只需知道函数的模代数表示式, 就能按式中各 项变量指数 (地址)填图, 无需改写成式 (1) 的形式; (2) 各变量在图中的方位不是唯一的, 只 需将填图量按地址作相应的重新编排即可.

例 1. 二变量三值函数

$$
F_{1}\left(X_{1}, X_{2}\right)=2 \oplus X_{2} \oplus 2 X_{2}^{2} \oplus X_{1} \oplus 2 X_{1} X_{2} \oplus X_{1} X_{2}^{2}
$$

的三值 $b_{i}$ 系数图如图 2(a) 或 (b) 所示.

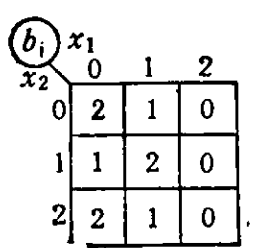

(a)

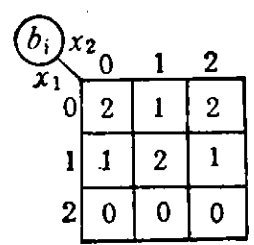

(b)

$$
\begin{aligned}
& c_{k_{0}} c_{k_{1}} c_{k_{2}} \\
& x_{1}: \begin{array}{llll}
1 & 2 & 0 & \left(f_{1}\right)
\end{array} \\
& \begin{array}{llll}
\multicolumn{3}{c}{d_{k_{0}} d_{k_{1}}} & d_{k 2} \\
x_{2}: & 2 & 1 & 2
\end{array} \quad\left(f_{23}\right)
\end{aligned}
$$

(c)

图 2 函数 $F_{1}$ 的三值 $b_{i}$ 图

\section{二、应用三值 $b_{i}$ 国对逻辑函数图形综合}

在三值 $b_{j}$ 系数图上综合逻辑函数，既不同于二值的情形 ${ }^{[2]}$, 也不同于三值 $K$ 图 $^{[4]}$, 它的基 础是单变量函数的化简,方法是图形分解.

对三值函数的规范 RM 展开式, 可进行如下的分解改写:

$$
\begin{gathered}
f\left(X_{1}, X_{2}\right)=b_{0} \oplus b_{1} X_{2} \oplus b_{2} X_{2}^{2} \oplus b_{3} X_{1} \oplus \cdots \oplus b_{8} X_{1}^{2} X_{2}^{2} \\
-\sum_{k=1}^{m} \oplus\left(c_{k 0} \oplus c_{k_{1}} X_{1} \oplus c_{k_{2}} X_{1}^{2}\right)\left(d_{k 0} \oplus d_{k_{1}} X_{2} \oplus d_{k_{2}} X_{2}^{2}\right), \\
f\left(X_{1}, X_{2}, X_{3}\right)=b_{0} \oplus b_{1} X_{3} \oplus b_{2} X_{3}^{2} \oplus b_{3} X_{2} \oplus \cdots \oplus b_{20} X_{1}^{2} X_{2}^{2} X_{3}^{2} \\
-\sum_{k=1}^{l} \oplus\left(c_{k 0} \oplus c_{k_{1}} X_{1} \oplus c_{k_{2}} X_{1}^{2}\right)\left(d_{k 0} \oplus d_{k 1} X_{2} \oplus d_{k_{2}} X_{2}^{2}\right)\left(e_{k 0} \oplus e_{k 1} X_{3} \oplus e_{k 2} X_{3}^{2}\right),
\end{gathered}
$$

\begin{tabular}{|c|c|c|c|c|c|c|c|c|c|c|}
\hline \multirow{2}{*}{ 函 } & \multirow{2}{*}{ 数 } & \multicolumn{3}{|c|}{ 系 数 组 } & \multirow{2}{*}{ 简 } & \multirow{2}{*}{ 函 } & \multicolumn{3}{|c|}{ 系 数 组 } & \multirow{2}{*}{ 简 } \\
\hline & & $b_{0}$ & $b_{1}$ & $b_{2}$ & & & $b_{0}$ & $b_{1}$ & $b_{2}$ & \\
\hline & 6 & 0 & 0 & 0 & 0 & $f_{14}$ & 1 & 1 & 2 & $2 \oplus 2 \tilde{x}^{2}$ \\
\hline & 1 & 0 & 0 & 1 & $x^{2}$ & $t_{1}$, & 1 & 2 & 0 & $2 y$ \\
\hline & 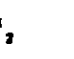 & 0 & 0 & 2 & $2 x^{\prime}$ & 1.0 & 1 & 2 & 1 & $\tilde{\mathbf{x}}^{2}$ \\
\hline & 3 & 0 & 1 & 0 & $x$ & $t_{11}$ & 1 & 2 & 2 & $2 \oplus 2 \vec{x}^{2}$ \\
\hline & 4 & 0 & 1 & 1 & $2 \oplus \tilde{x}^{2}$ & $f_{18}$ & 2 & 0 & 0 & 2 \\
\hline & 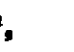 & 0 & 1 & 2 & $1 \oplus 2 \tilde{x}^{2}$ & $t_{1}$ & 2 & 0 & 1 & $2 \oplus x^{2}$ \\
\hline & 6 & 0 & 2 & 0 & $2 x$ & $t_{20}$ & 2 & 0 & 2 & $2 \oplus 2 x^{2}$ \\
\hline & , & o & 2 & 1 & $2 \oplus \tilde{x}^{2}$ & $f_{21}$ & 2 & 1 & 0 & 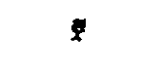 \\
\hline & 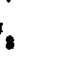 & 0 & 2 & 2 & $1 \oplus 2 x^{2}$ & $t_{22}$ & 2 & 1 & 1 & $1 \oplus x^{2}$ \\
\hline & . & 1 & 0 & 0 & 1 & $f_{23}$ & 2 & 1 & 2 & $2 \tilde{\boldsymbol{x}}^{\mathbf{2}}$ \\
\hline & 10 & 1 & 0 & 1 & $1 \oplus x^{2}$ & $t_{24}$ & 2 & 2 & 0 & $2 \tilde{x}$ \\
\hline & 11 & 1 & 0 & 2 & $1 \oplus 2 x^{2}$ & $f_{2}$ & 2 & 2 & 1 & $1 \oplus \tilde{x}^{2}$ \\
\hline & 112 & 1 & 1 & 0 & $\tilde{\boldsymbol{x}}$ & $t_{20}$ & 2 & 2 & 2 & $2 x^{2}$ \\
\hline & 13 & 1 & 1 & 1 & $x^{2}$ & & & & & \\
\hline
\end{tabular}

表 1 单变量三值函数

注: $\hat{x}=1 \oplus x, x=2 \oplus x$. 
式 (3) 和 (4) 表明, 任一函数的原始 $b_{i}$ 图可按模 3 加的法则, 分解成几个相同维数、相同菒 相同地址的分 $b_{i}$ 图，圆括号中各系数的模 3 积等于分 $b_{i}$ 图中相应方格的填人量. 而每个 圆括号内则是单变量函数。

利用变量的极性变换 ${ }^{[3]}$, 表 1 给出 27 种单变量函数的简式。

对于例 1 的函数 $F_{1}$, 它的原始 $b_{i}$ 图较简单,可理解成图 2(c). 因此可得

$$
F_{1}\left(X_{1}, X_{2}\right)=f_{15}\left(X_{1}\right) f_{23}\left(X_{2}\right)=\widetilde{X}_{1} \widetilde{X}_{2}^{2}
$$

当然, 并非所有函数的 $b_{j}$ 图都似图 2 这样简单. 我们称似图 2 那样, 能用单一积项表示 的三值 $b_{i}$ 图为简单 $b_{i}$ 图. 否则, 称为复杂 $b_{i}$ 图.

例 2. 二变量三值函数

$$
F_{2}\left(X_{1}, X_{2}\right)=X_{2} \oplus X_{2}^{2} \oplus X_{1} \oplus 2 X_{1} X_{2} \oplus X_{1} X_{2}^{2} \oplus X_{1}^{2} \oplus 2 X_{1}^{2} X_{2} \oplus X_{1}^{2} X_{2}^{2},
$$

该函数的三值 $b_{i}$ 图-图 3 左边是复杂 $b_{i}$ 图. 利用模 3 加法则, 可将该图分解成图 3 右边, 使

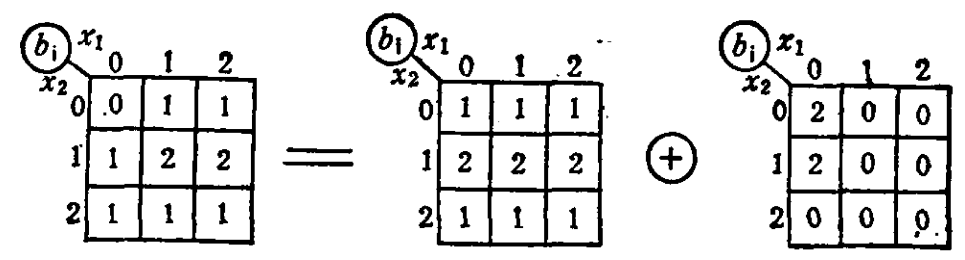

图 $3 F_{2}$ 的三值 $b_{1}$ 图及分解

每个分 $b_{i}$ 图均为简单 $b_{i}$ 图, 从而得出

$$
F_{2}\left(X_{1}, X_{2}\right)=f_{13}\left(X_{1}\right) f_{16}\left(X_{2}\right) \oplus f_{9}\left(X_{1}\right) f_{78}\left(X_{2}\right)=\widetilde{X}_{1}^{2} \widetilde{X}_{2}^{2} \oplus 2 \widetilde{X}_{2},
$$

与文献 [5] 矩阵运算的结果完全一致.

至此,可归纳应用三值 $b_{i}$ 图图形综合方法如下:

(1) 绘制函数的三值 $b_{j}$ 图;

（2）利用表 1 , 分析该图是否是简单 $b_{i}$ 图. 是,转到 (4); 不是,进行下一步 (3);

（3）将复杂 $b_{i}$ 图分解成若干个简单 $b_{i}$ 图之和,要求分图的个数尽量少;

(4) 写出各简单 $b_{i}$ 图的函数简式;

（5）将各分图的简式相加一模 3 和,即得原函数的简式.

\section{三、应用三值 $\boldsymbol{b}_{\text {; }}$ 图选择摸块设计最佳方案}

在多值逻辑研究中,以单一模块为细胞的树型结构函数实现电路,具有简单、实用等优点. Tokmen 和 Hurst 提出采用通用逻辑组件 ${ }^{[6]}$ :

$$
U_{t}=\alpha \oplus \beta X \oplus r X^{2},
$$

实现三值逻辑函数。其基本方法是对函数的规范 RM 展开式括公因子，确定控制输人变量级 序及每个 $U_{f}$ 模块的输入量。对 $n$ 变量三值函数, 设变量 $X_{n}$ 被取作第一级控制输入变量, 则 对式 (1) 括公因子得

$$
\begin{aligned}
f\left(X_{1}, \cdots, X_{n}\right)= & \left(b_{0} \oplus b_{1} X_{n} \oplus b_{2} X_{n}^{2}\right) \oplus\left(b_{3} \oplus b_{4} X_{n} \oplus b_{5} X_{n}^{2}\right) X_{n-1} \oplus \cdots \\
& \oplus\left(b_{3^{n}-3} \oplus b_{3^{n}-2} X_{n} \oplus b_{3^{n}-1} X_{n}^{2}\right) X_{1}^{2} \cdots X_{n-1}^{2},
\end{aligned}
$$

式 (5) 中每个圆括号中的三系数, 即是第一级各 $U_{f}$ 模块的三输入. 对 $U_{f}$ 电路化简的工作 是: 
(1) $U_{f}$ 输出为常数,条件是 $\beta=\gamma=0$;

(2) $U_{f}$ 输出为原控制变量,条件是 $\beta=1, \alpha=\gamma=0$;

(3) 二个以上 $U$, 的输出相同,条件是 $\alpha, \beta, \gamma$ 对应相等.

简化 $U_{f}$ 结构电路，即用最少的 $U_{f}$ 模块实现给定函数。上述 (1) 和 (2), 不需用 $U_{f}$; 上述 (3)，可省用 $U_{t}$. 这与第一级控制变量的选取,关系甚大. 文献 [6] 考虑到这些，但用的是代 数法,即需对给定函数作式 (5) 形式的改写,有几个变量就需改写几次, 这显然是麻烦的. 且 观察、分析简化条件甚不方便。

观察图 1, 式 (5) 中各圆括号内三系数在三值 $b_{j}$ 图中恰作规则排列，比较哪个变量为第 一级控制输入较宜非常方便, 寻求 $U_{f}$ 简化条件非常直观。

例 3. 用 $U_{t}$ 模块实现三值函数

$$
F_{3}\left(X_{1}, X_{2}\right)=2 \oplus 2 X_{2}^{2} \oplus X_{1} X_{2} \oplus X_{1} X_{2}^{2} \oplus X_{1}^{2} X_{2}^{2},
$$

$F_{3}$ 的三值 $b_{i}$ 图如图 4(a) 所示. 由图直接可知, 以 $X_{2}$ 为第一级控制输入, 需用 4 个 $U_{f}$ 模块; 选 $X_{1}$ 只需用 2 个 $U_{t}$ 模块. 当变量数增多时，利用三值 $b_{i}$ 图寻求最佳实现方案的优点更明 显。

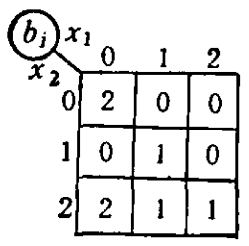

(a)

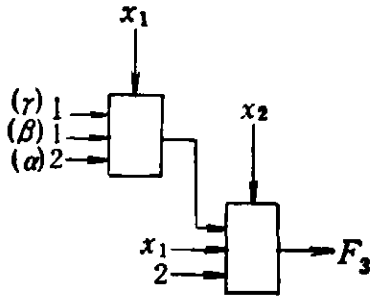

(b)

图 4

(a) $F_{3}$ 的三值 $b_{1}$ 图；（b) $F_{3}$ 的 $U_{1}$ 模块实现

由上可知, 三值 $b_{i}$ 系数图的引人，给三值逻辑函数的综合提供了一个有效的图形工具. 当然,如同其它的图形方法一样,需要设计者一定的技巧和受变最数不能太多的限制. 我们的 方法在变量数 $\leqslant 4$ 的情况下是有效的.

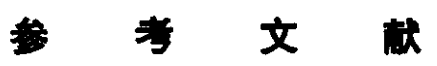

[1] Karnaugh, M., AlEE Trans. Commun. Electron, 9(1953), 593-599.

[ 2] Wu, X., Chen, X. \& Hurst, S. L., IEE Proc. Pz. E, 129 (1982), 15-20.

[3] Chen, X., Computers \& Electrical Engineering, 9 (1982), 167-180.

[4] 吴训威、左江凯, 科技通报, 2(1986)，2: 3，11-13.

[ 5 ] Chen, X. \& Wu, X.. Proc. 13th ISMVL, 1983, 424-429.

[6] Tokmen, V. H. \& Hurst, S. L., Proc. 9th ISMVL, 1979, 248-256. 